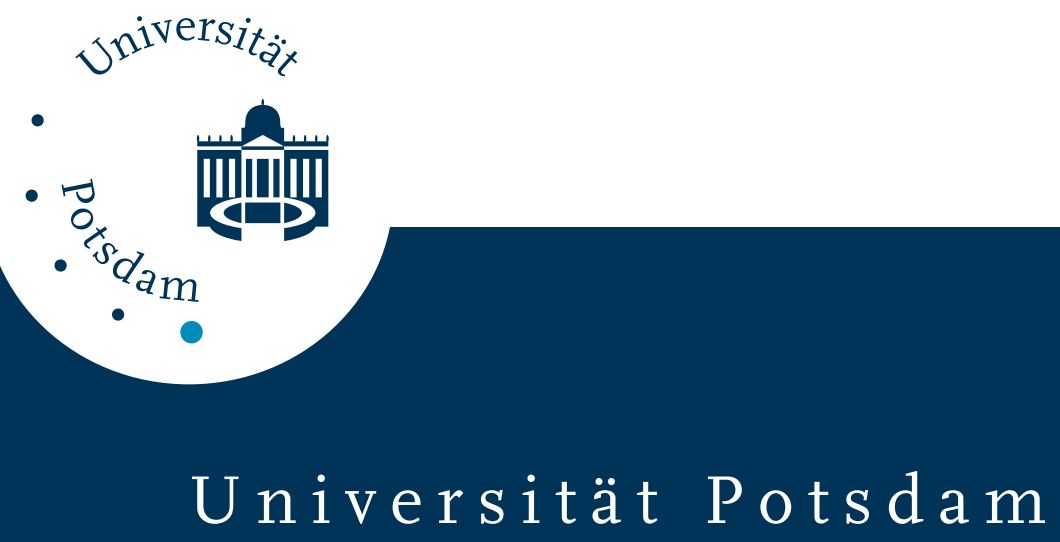

Boris Schäfer-Bung, Mathias Nest

\title{
Correlated dynamics of electrons with reduced two-electron density matrices
}

first published in:

Physical Review A 78 (2008), 012512, ISSN 1094-1622,

DOI 10.1103/PhysRevA.78.012512

Postprint published at the Institutional Repository of the Potsdam University:

In: Postprints der Universität Potsdam

Mathematisch-Naturwissenschaftliche Reihe ; 100

http://opus.kobv.de/ubp/volltexte/2010/4177/

http://nbn-resolving.de/urn:nbn:de:kobv:517-opus-41774

Postprints der Universität Potsdam

Mathematisch-Naturwissenschaftliche Reihe ; 100 


\title{
Correlated dynamics of electrons with reduced two-electron density matrices
}

\author{
B. Schäfer-Bung and M. Nest \\ Institut für Chemie, Universität Potsdam, Karl-Liebknecht-Straße 24-25, D-14476 Potsdam, Germany
}

(Received 12 March 2008; published 28 July 2008)

\begin{abstract}
We present an approach to the correlated dynamics of many-electron systems. We show, that the twoelectron reduced density matrix $(2 \mathrm{RDM})$ can provide a suitable description of the real time evolution of a system. To achieve this, the hierarchy of equations of motion must be truncated in a practical way. Also, the computational effort, given that the $2 \mathrm{RDM}$ is represented by products of two-electron determinants, is discussed, and numerical model calculations are presented.
\end{abstract}

DOI: 10.1103/PhysRevA.78.012512

PACS number(s): 31.10.+z, 31.15.-p, 71.10.-w

\section{INTRODUCTION}

In recent years there has been a growing interest in the correlated dynamics of systems of many electrons. Especially the tremendous progress in experimental technology, which allows the observation of the movement of electrons on their natural time scale of femtoseconds $\left(1 \mathrm{fs}=10^{-15} \mathrm{~s}\right)$ or less, has contributed to this development [1-8]. At the same time, theoreticians have approached the problem in two different ways: On the one hand, there are density functional (DFT) based methods [9-12], in which the three-dimensional electron density is propagated. DFT can treat rather large systems, but they provide only qualitative results, because the functionals are usually made to reproduce ground-state properties. Wave-function-based methods for correlated electron dynamics are much more accurate, but can treat only much smaller systems [13-21]. The reason for this is, that wave functions are $3 N$ dimensional, with $N$ being the number of electrons. Thus, the computational effort for accurate solutions becomes prohibitively large with increasing $N$.

In this paper we suggest using the reduced two-electron density matrix (2RDM) instead, because it combines attractive features from both worlds. The idea to establish a quantum chemistry based on the 2RDM goes back to Löwdin, Meyer, and Coulson [22-25]. They realized that the functional that calculates the electronic energy from a $2 \mathrm{RDM}$ is analytically known, and easy to evaluate. (See the next section for details.) At the same time, the 2RDM has the size of just a four-electron wave function, independent of the actual number $N$ of electrons. The fact that a comparatively small object allows the calculation of the exact energy (or FCI energy, full configuration interaction) has led to a lot of time and effort being invested into this approach. However, it turned out to be surprisingly difficult to turn this ansatz into a competitive tool for electronic structure calculations. This is due to the fact that a naive variation of the $2 R D M$ in search for the lowest energy produces a result well below the ground-state energy, because there are more 2RDMs than can be derived from a proper, antisymmetric $N$-electron wave function. Therefore, the naive variational approach leads in general to a nonfermionic ground state. Those $2 \mathrm{RDMs}$ that correspond to a true fermionic ground state are called $N$ representable [26]. But to impose $N$ representability during variation has long been a problem which scaled as $K^{16}$, where $K$ is the number of spatial one-electron basis func- tions, and could be improved to a still expensive $K^{6}$ only recently [27]. Fortunately, this problem does not translate one-to-one to the solution of time-dependent problems, if the initial state of the propagation is derived from a wave function, thus ensuring $N$ representability. It is the subject of this paper to discuss the possibilities and problems of electron dynamics using the 2RDM.

Two questions will be at the center of this study: The first concerns the equation of motion. The time evolution of density matrices of correlated particles is governed by a hierarchy of equations [28]. In other words, the time derivative of the 1RDM depends on the 2RDM, the derivative of the 2RDM depends on the $3 \mathrm{RDM}$, and so on. The truncation of this hierarchy is a necessary approximation that must be introduced for practical calculations. The second question concerns the representation of the 2RDM. Even if only $K=100$ spatial basis function are employed, then the density matrix requires 24 GB memory, in the product basis. Obviously, a more efficient approach is required. Here, an ansatz with products of two-electron determinants suggests itself in the context of quantum chemistry.

The paper is organized as follows. The next section describes the theory, both for the representation of the 2RDM, as well as for the truncation of the hierarchy of equations of motion. Section III will provide numerical examples, and discuss the performance of the method. Atomic units will be used throughout this paper, if not stated otherwise. (The symbol $E_{h}$ denotes the Hartree energy, where $1 E_{h}$ $=27.21138 \mathrm{eV}$.)

\section{THEORY}

We begin the theory section of this paper by describing the representation of the $2 \mathrm{RDM}, \hat{\rho}^{(2)}$, by means of products of two-electron Slater determinants. Section II B will rederive briefly the equation of motion, and Sec. II C discusses our ansatz for the truncation of the hierarchy of equations of motion.

\section{A. Representation of $\hat{\boldsymbol{\rho}}^{(2)}$}

A representation by means of determinants requires the choice of orbitals, from which the determinants are built. We follow here the standard quantum chemical approach, and choose Hartree-Fock orbitals. For a given number $K$ of basis 
functions or grid points, $2 K$ Hartree-Fock spin orbitals are obtained. We enumerate them by small italic letters $j$, and denote $N$-electron determinants by

$$
\left|\operatorname{det}\left(J^{N}\right)\right\rangle=\left|\operatorname{det}\left(j_{1} \cdots j_{N}\right)\right\rangle .
$$

Every ordered $N$-tuple $J^{N}$ defines uniquely a determinant, so that arbitrary electronic wave functions can be represented as

$$
|\Psi\rangle=\sum_{J^{N}}^{\text {ord }} A_{J^{N}}\left|\operatorname{det}\left(J^{N}\right)\right\rangle .
$$

Note that the summation of all ordered $N$-tuples corresponds to a FCI state, if all $2 K$ orbitals are used, or a complete active space configuration interaction (CASCI) state, if a smaller number of orbitals is used. Correspondingly, we expand $\hat{\rho}^{(2)}$ with the help of two-electron determinants $\left|\operatorname{det}\left(J^{2}\right)\right\rangle$,

$$
\begin{aligned}
\hat{\rho}^{(2)}\left(1,2 ; 1^{\prime}, 2^{\prime}\right)= & \sum_{J^{2}, K^{2}}^{\text {ord }} \frac{1}{(2 !)^{2}} B_{J^{2}, K^{2}}\left|\operatorname{det}\left(J^{2}\right)(1,2)\right\rangle \\
& \times\left\langle\operatorname{det}\left(K^{2}\right)\left(1^{\prime}, 2^{\prime}\right)\right|,
\end{aligned}
$$

where $i=x_{i}=\left(r_{i}, s_{i}\right)$ describes a combined position and spin variable. From this, the reduced one-electron density matrix is obtained by tracing out electron coordinate 2 ,

$$
\hat{\boldsymbol{\rho}}^{(1)}\left(1 ; 1^{\prime}\right)=\operatorname{Tr}_{2}\left\{\hat{\boldsymbol{\rho}}^{(2)}\left(1,2 ; 1^{\prime}, 2\right)\right\}=\sum_{j, k} D_{j, k}|j(1)\rangle\left\langle k\left(1^{\prime}\right)\right| .
$$

Equations (3) and (4) serve also to define the coefficients $B_{J^{2}, K^{2}}$ and $D_{j, k}$.

Practically, solving the time-dependent Schrödinger equation means to solve an initial value problem, with the initial state often being the ground state. Therefore, we start from a wave function $\Psi_{0}$, defined by the coefficients $A_{J^{N}}$ in Eq. (2), and calculate the coefficients $B_{J^{2}, K^{2}}$ of the 2RDM with the help of the so-called double-hole functions, $\Psi_{J^{2}}^{\mathrm{DHF}}$ :

$$
|\Psi\rangle=\frac{1}{2 !} \sum_{J^{2}}^{\text {ord }}\left|\operatorname{det}\left(J^{2}\right)\right\rangle \underbrace{\left\langle\operatorname{det}\left(J^{2}\right) \mid \Psi\right\rangle}_{\Psi_{J^{2}}^{\mathrm{DHF}}},
$$

and

$$
\hat{\rho}^{(2)}=\sum_{J^{2}, L^{2}}^{\text {ord }} \frac{1}{(2 !)^{2}}\langle\underbrace{\Psi_{L^{2}}^{\mathrm{DHF}} \mid \Psi_{J^{2}}^{\mathrm{DHF}}}_{B_{J^{2}, L^{2}}}\rangle\left|\operatorname{det}\left(J^{2}\right)\right\rangle\left\langle\operatorname{det}\left(L^{2}\right)\right| .
$$

This leads to the following expression for the initialization of the 2RDM (see the Appendix for details):

$$
B_{J^{2}, L^{2}}=(N-2) !(2 !)^{2} \sum_{K^{N-2}}^{\text {ord }}\left(A_{L^{2} K^{N-2}}\right)^{*} A_{J^{2} K^{N-2}} .
$$

\section{B. Equations of motion}

The time evolution of the $N$-electron wave function is governed by the Hamiltonian

$$
\hat{H}^{(N)}=\sum_{i=1}^{N}\left[\hat{T}(i)+\hat{V}_{e n}(i)\right]+\sum_{i>j}^{N} \hat{V}_{e e}(i, j),
$$

with kinetic energy $\hat{T}$, electron-nuclear attraction $\hat{V}_{\text {en }}$, and electron-electron repulsion $\hat{V}_{e e}$. As mentioned in the introduction, it is possible to calculate the energy expectation value from the 2RDM alone, because the Hamiltonian contains only up to two-particle interactions,

$$
\begin{aligned}
\left\langle\hat{H}^{(N)}\right\rangle= & N \operatorname{Tr}_{1}\left\{\left[\hat{T}(1)+\hat{V}_{e n}(1)\right] \hat{\rho}^{(1)}\right\} \\
& +\frac{N(N-1)}{2} \operatorname{Tr}_{1,2}\left[\hat{V}_{e e}(1,2) \hat{\rho}^{(2)}\right] .
\end{aligned}
$$

The time evolution of the 2RDM is most easily derived from the NRDM,

$$
\hat{\rho}^{(N)}=|\Psi\rangle\langle\Psi|,
$$

which is governed by the closed-system Liouville-von Neumann equation

$$
\dot{\hat{\rho}}^{(N)}=-i\left[\hat{H}^{(N)}, \hat{\rho}^{(N)}\right] .
$$

The time derivative of $\hat{\rho}^{(2)}$ is then, in principle, obtained by tracing out electrons 3 through $N$,

$$
\dot{\hat{\rho}}^{(2)}=-i \operatorname{Tr}_{3 \cdots N}\left\{\left[\hat{H}^{(N)}, \hat{\rho}^{(N)}\right]\right\} .
$$

Practically, the elimination of the electrons 3 through $N$ is problematic. The first step is to divide the Hamiltonian $\hat{H}^{(N)}$ into three parts: Part one contains only the first two electron coordinates and momenta, part two depends only on electrons 3 to $N$, and part three contains the coupling,

$$
\begin{aligned}
\dot{\hat{\rho}}^{(2)}= & -i \operatorname{Tr}_{3 \cdots N}\left\{\left[\hat{H}^{(2)}, \hat{\boldsymbol{\rho}}^{(N)}\right]\right\}-i \sum_{i=3}^{N} \operatorname{Tr}_{3 \cdots N} \\
& \times\left[\left(\hat{T}(i)+\hat{V}_{e n}(i)+\sum_{j=i+1}^{N} \hat{V}_{e e}(i, j), \hat{\boldsymbol{\rho}}^{(N)}\right)\right] \\
& -i \sum_{j=3}^{N} \operatorname{Tr}_{3 \cdots N}\left\{\left[\hat{V}_{e e}(1, j)+\hat{V}_{e e}(2, j), \hat{\boldsymbol{\rho}}^{(N)}\right]\right\} \\
= & \mathcal{L}_{1}\left[\hat{\boldsymbol{\rho}}^{(N)}\right]+\mathcal{L}_{2}\left[\hat{\boldsymbol{\rho}}^{(N)}\right]+\mathcal{L}_{3}\left[\hat{\boldsymbol{\rho}}^{(N)}\right] .
\end{aligned}
$$

The first term can be evaluated easily to give

$$
\mathcal{L}_{1}\left[\hat{\rho}^{(N)}\right]=-i\left[\hat{H}^{(2)}, \hat{\rho}^{(2)}\right]
$$

The second term, $\mathcal{L}_{2}\left[\hat{\rho}^{(N)}\right]$, is zero. The third term survives, and can be simplified as follows. Due to the indistinguishability of the electrons, each of the $(N-2)$ summands of $\mathcal{L}_{3}$ labeled by the electron $j$ provides the same contribution, which can be derived by performing the trace on all electrons but $j$ (using the commutativity in partial traces), 


$$
\begin{aligned}
\mathcal{L}_{3}\left[\hat{\rho}^{(N)}\right] & =-i \sum_{j=3}^{N} \operatorname{Tr}_{3 \cdots N}\left\{\left[\hat{V}_{e e}(1, j)+\hat{V}_{e e}(2, j), \hat{\rho}^{(N)}\right]\right\} \\
& =-i(N-2) \operatorname{Tr}_{3}\left\{\left[\hat{V}_{e e}(1,3)+\hat{V}_{e e}(2,3), \hat{\rho}^{(3)}\right]\right\} .
\end{aligned}
$$

Thus, the time derivative of $\hat{\rho}^{(2)}$ depends on $\hat{\rho}^{(3)}$. If $\hat{\rho}^{(3)}$ should also be determined by the equations of motion (EOM) one would have to know $\hat{\rho}^{(4)}$, and so on. This leads to a hierarchy of EOMs [28] where $\dot{\hat{\rho}}^{(M)}$ depends on $\hat{\rho}^{(M)}$ and $\hat{\rho}^{(M+1)}$ in analogy to the Bogolyubov-Born-Green-KirkwoodYvon (BBGKY) hierarchy in statistical mechanics [29]. Summarizing, the equation of motion is given by

$$
\dot{\hat{\rho}}^{(2)}=-i\left[\hat{H}^{(2)}, \hat{\rho}^{(2)}\right]-i(N-2) \operatorname{Tr}_{3}\left\{\left[\hat{V}_{e e}(1,3)+\hat{V}_{e e}(2,3), \hat{\rho}^{(3)}\right]\right\}
$$

$$
=\dot{\hat{\rho}}^{(2), \text { pair }}+\dot{\hat{\rho}}^{(2), \text { add }} .
$$

Because the dependence on $\hat{\rho}^{(3)}$ cannot be eliminated, we will outline a simple reconstruction scheme in the next section. Equation (18) can be recast into an equation for the expansion coefficients of the 2RDM, to give

$$
\dot{B}_{J^{2}, K^{2}}=\left\langle\operatorname{det}\left(J^{2}\right)\left|\dot{\hat{\rho}}^{(2)}\right| \operatorname{det}\left(K^{2}\right)\right\rangle=\dot{B}_{J^{2}, K^{2}}^{(\text {pair }}+\dot{B}_{J^{2}, K^{2}}^{(\text {add }} .
$$

The first part describes an uncoupled electron pair and can be expanded into

$$
\dot{B}_{J^{2}, K^{2}}^{\text {pair }}=-\frac{i}{2 !} \sum_{L^{2}}^{\text {ord }} H_{J^{2}, L^{2}}^{(2)} B_{L^{2}, K^{2}}-B_{J^{2}, L^{2}} H_{L^{2}, K^{2}}^{(2)},
$$

where the $H_{J^{2}, L^{2}}^{(2)}$ are matrix elements of the two-electron Hamiltonian, $\left\langle\operatorname{det}\left(J^{2}\right)\left|\hat{H}^{(2)}\right| \operatorname{det}\left(L^{2}\right)\right\rangle$ which can be evaluated easily and efficiently by means of Slater-Condon rules [30]. The second part $\dot{B}_{J^{2}, K^{2}}^{(\text {add) }}$ represents the electron-electron coupling between this electron pair and all other electrons.

\section{Reconstruction of $\hat{\boldsymbol{\rho}}^{(\mathbf{3})}$}

Several groups have made suggestions for reconstruction functionals for the $3 \mathrm{RDM}$ or the $4 \mathrm{RDM}$, with various degrees of sophistication [28,31-34]. In this paper we will deal with the most simple approach, namely, we reconstruct $\hat{\rho}^{(3)}$ with a Grassmann product $[31,35]$ of the $2 \mathrm{RDM}$ and $1 \mathrm{RDM}$,

$$
\hat{\rho}^{(3)} \approx \hat{\rho}_{r c}^{(3)}=\frac{3 N}{N-2} \hat{A}\left\{\hat{\rho}^{(1)} \otimes \hat{\rho}^{(2)}\right\} \hat{A}^{\dagger},
$$

where the prefactor is included for reasons of normalization, $\hat{A}$ represents an idempotent antisymmetrizer for three particle wave functions,

$$
\hat{A}=\frac{1}{3 !}\left(\hat{I}-\hat{P}_{12}-\hat{P}_{13}-\hat{P}_{23}+\hat{P}_{23} \hat{P}_{12}+\hat{P}_{23} \hat{P}_{13}\right),
$$

and $\hat{P}_{i j}$ interchanges the $i$ th and $j$ th electron. Insertion of our representations of the one- and two-electron density matrices and rearranging gives

$$
\begin{aligned}
\hat{\rho}_{r c}^{(3)}= & \frac{3 N}{(N-2)} \sum_{J^{3}, K^{3}}^{\text {ord }} \frac{1}{(3 !)^{2}} \sum_{i, l=1}^{3}(-1)^{i+l} D_{j_{i}, k_{l}} B_{J_{i}^{2}}, K_{l}^{2}\left|\operatorname{det}\left(J^{3}\right)\right\rangle \\
& \times\left\langle\operatorname{det}\left(K^{3}\right)\right|
\end{aligned}
$$

$\left(J^{3}\right.$ is the ordered composition of $j_{i}$ and $\left.J_{i}^{2}\right)$ which is our working equation for the reconstruction for the examples in Sec. III.

\section{EXAMPLES AND DISCUSSION}

In this section we discuss the performance of configuration interaction- (CI) based electron dynamics using the 2RDM and the example of a one-dimensional $\mathrm{Be}$ atom. It is the smallest possible closed shell system, where the 2RDM represents a number of electrons larger than 2, and it is at the same time possible to solve it in a numerically exact way. The purpose of these examples are to demonstrate the level of accuracy that can be achieved by the reconstruction scheme for $\hat{\rho}^{(3)}$ given in Eq. (21). As outlined in the preceding section, the reconstruction can provide only a certain fraction of the correlation. Therefore, we will present two examples, based on configuration interaction with single excitations (CIS) and CASCI selections for the $\left|\operatorname{det}\left(J^{N}\right)\right\rangle$ basis functions. In both cases, we have restricted ourselves to $N_{\mathrm{SO}}=20$ spin orbitals, which gives converged results as can be checked by the time-dependent HF orbital populations. In traditional quantum chemical notation the active space of CASCI would then be denoted $(4,10)$, for four electrons in 10 spatial orbitals.

In order to avoid the Coulomb singularity, we screen the potentials as usual $[13,16,36]$ in one-dimensional model systems in the following way:

$$
\begin{gathered}
V_{e n}\left(r_{i}\right)=-\frac{4}{\sqrt{r_{i}^{2}+c}}, \\
V_{e e}\left(r_{i}, r_{j}\right)=\frac{1}{\sqrt{\left(r_{i}-r_{j}\right)^{2}+c}} .
\end{gathered}
$$

The screening constant was chosen to be $c=1 a_{0}^{2}$.

Next, we determined the Hartree-Fock orbitals of the system on a grid with $K=64$ grid points. These are used to construct the two-electron determinants for the representation of the 2RDM, and serve also in our wave-function-based benchmark calculations.

Initially, at time $t=0$, the system is assumed to be in its ground state. In the case of CIS the ground-state wave function $\Psi_{\mathrm{GS}}^{\mathrm{CIS}}$ is the Hartree-Fock determinant, by Brillouin's theorem. In the case of CASCI the Hamiltonian matrix is represented by all 4845 determinants in the active space, and then diagonalized, yielding $\Psi_{\mathrm{GS}}^{\mathrm{CASCI}}$. On both levels of theory the corresponding ground-state $2 \mathrm{RDM}$ is then determined via the double-hole function approach described in Sec. II.

The dynamics of the four-electron system is initiated by an ultrashort laser pulse of $t_{w}=2$ fs duration, a carrier frequency of $\omega=0.5 E_{h} / \hbar$, and a sine-square envelope 


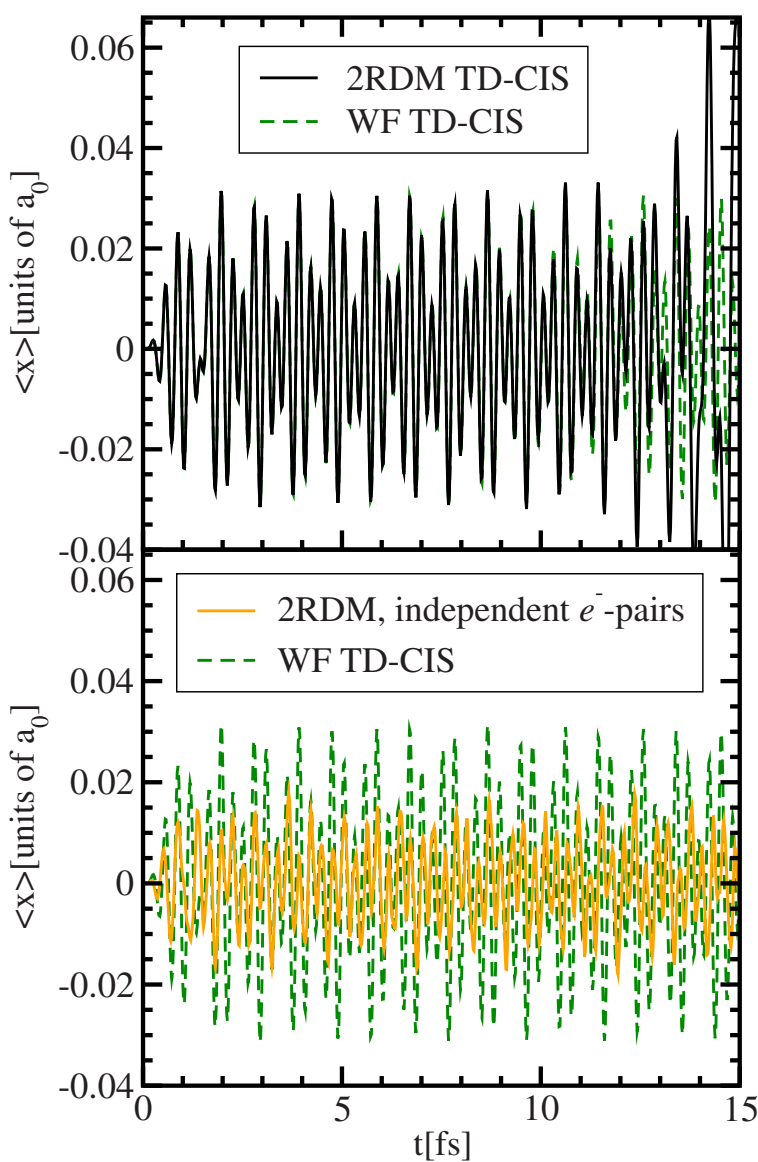

FIG. 1. (Color online) Comparison of the time-dependent position expectation values for a 2 RDM calculation (black solid line), 2RDM approach using independent electron pairs (orange solid line), and wave function computation (green dashed line). All calculations are restricted to a CIS orbital space.

$$
\vec{E}(t)=\vec{E}_{0} \sin ^{2}\left(\pi t / t_{w}\right) \cos (\omega t) \quad t \in\left[0, t_{w}\right],
$$

which is coupled to the electrons in the semiclassical dipole approximation. The ultrashort duration implies that the pulse is spectrally very broad, and populates several excited states. (The first excitation energy is $0.222 E_{h}$ for CIS and $0.210 E_{h}$ for CASCI.)

\section{A. Single excitations}

In this section we compare wave-function based timedependent CIS (TD-CIS) calculations with time-dependent 2RDM calculations, where the $2 \mathrm{RDM}$ and the reconstructed 3RDM are restricted to contain at most single excitations. At time $t=0$ the $2 \mathrm{RDM}$ is build from a Hartree-Fock (HF) ground state, and therefore the reconstruction of $\hat{\rho}^{(3)}$ is initially exact. The laser pulse then transfers population to excited states, and thus creates correlations, which can be reconstructed only in an approximate fashion in the 3RDM.

We begin with the time-dependent position expectation value. Figure 1 compares results for a comparatively low laser fluence of about $3.16 \times 10^{11} \mathrm{~W} / \mathrm{cm}^{2}$. For about the first $10 \mathrm{fs}$ the agreement between 2RDM (black solid line) and wave function calculations (green dashed line) is excellent, but later errors are accumulating, and the propagation gets out of control. Concurrent with this, we observe an exponential violation of the positive semidefiniteness, and thus of the $N$ representability.

Because the origin of the error is the reconstruction of the 3RDM, it is tempting to make the additional assumption of independent electron pairs. In this case, the coupling between electrons number 1 and 2 to electron number 3 is ignored, and thus the term $\dot{B}_{J^{2}, L^{2}}^{\text {(add) }}$ in Eq. (19) vanishes. Equivalently, one could say that we are neglecting the terms

$$
V_{e e}(1,3)+V_{e e}(1,4)+V_{e e}(2,3)+V_{e e}(2,4)
$$

in the Hamiltonian. Electrons 1 and 2 still are interacting. Figure 1 shows (orange solid line), that in this case the propagation remains stable, but a phase shift occurs. The stability of the propagations is supported by the finding that throughout the propagation all diagonal 2RDM remain positive, which identifies the reconstructed 3RDM clearly as the source of instability. Also, it should be noted that the EOM becomes nonlinear through the reconstruction of $\hat{\rho}^{(3)}$ which complicates the propagation further.

The corresponding energy expectation values are shown in the upper panel of Fig. 2. Again, the wave function and 2RDM results are in good agreement at early times, but later start to deviate exponentially. The energy expectation value for independent electron pairs (see orange solid line in Fig. $1)$, is shown separately in the inset, because it is shifted by about $2.25 E_{h}$ to lower energies. Such a low value can be obtained, because the modified Hamiltonian does not describe indistinguishable electrons anymore. However, we report these values here in order to illustrate the stability of the propagation in the absence of the nonlinear term.

In this first example, a comparatively small amount of energy was deposited in the electronic system. An increase of the laser fluence to $3.51 \times 10^{12} \mathrm{~W} / \mathrm{cm}^{2}$ and 3.16 $\times 10^{13} \mathrm{~W} / \mathrm{cm}^{2}$ causes higher excited determinants to become important, and thus pose a greater challenge to the reconstruction. The lower panel of Fig. 2 reports the energy expectation values for all three fluences and two methods. The lowest pair of curves (lowest fluence) is also shown in the upper panel. The deviations from the wave-function calculation begin for higher fluences at earlier times, and are more pronounced, as expected. As for the low fluence, we find that the energy expectation value is a much more sensitive probe of the quality of the propagation, than the position expectation value. The latter is generally well reproduced for about 10 fs.

In contrast to the independent pair approximation, the positivity of the diagonal $2 \mathrm{RDM}$ elements is violated within the first fs and the mean value of the most negative matrix element is rising while the laser field is on. Later, these elements oscillate around a fixed value which is approximately proportional to the laser intensity. After $10 \mathrm{fs}$, the most negative 2RDM elements start rising subtly but continuously and pass into an exponential increase around 15 fs. A method for avoiding the positivity violation was suggested [37] and could be implemented in the future. 


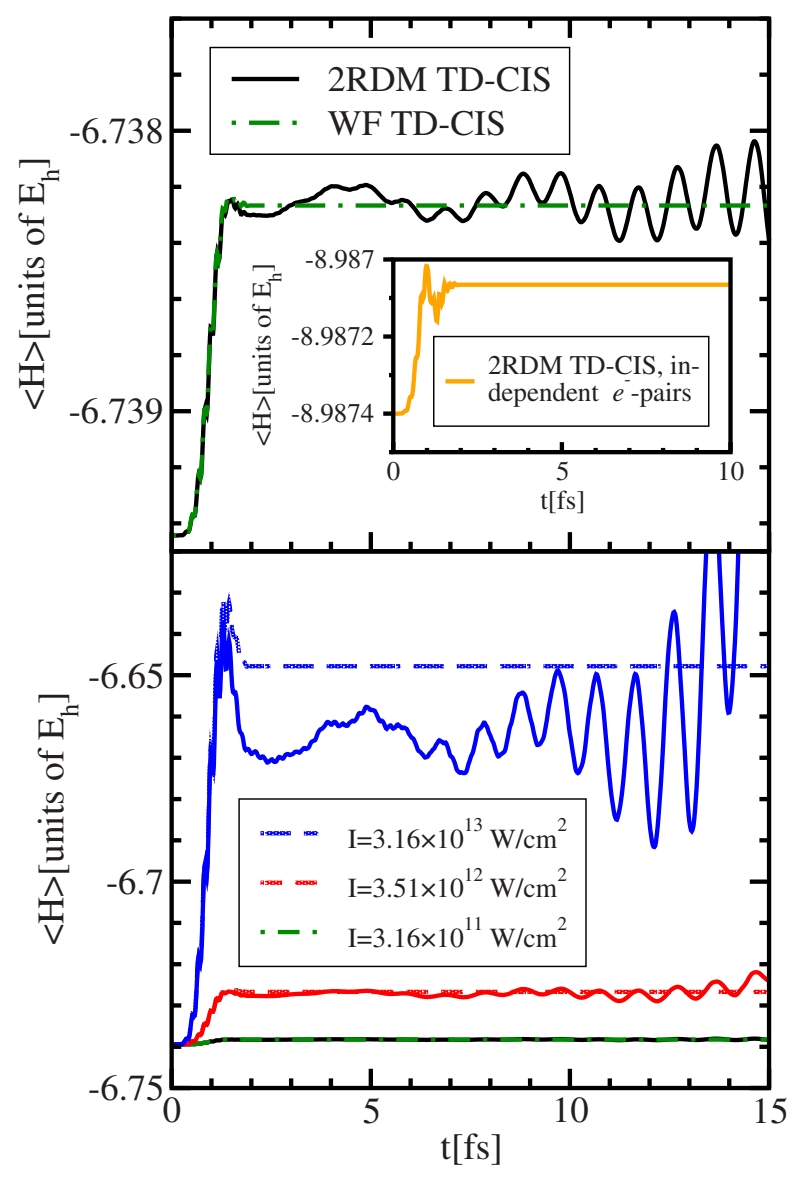

FIG. 2. (Color online) Time-dependent total energy for 2RDM and wave-function calculations. The upper panel shows 2RDM (solid line) and wave-function results (dashed-dotted line) for a laser fluence of $3.16 \times 10^{11} \mathrm{~W} / \mathrm{cm}^{2}$. The inset reflects the corresponding graph for the $2 \mathrm{RDM}$ independent electron pair approach. On the lower panel the 2RDM (solid lines) and corresponding wave-function calculations (dashed lines) are compared for different laser fluences (given in $\mathrm{W} / \mathrm{cm}^{2}$ ).

The restriction to singly excited determinants has been used, because the reconstruction is known to work better in this case. However, a 2RDM can ideally represent a full CI state, which is why we turn our attention now to the fully correlated dynamics.

\section{B. Complete active space}

From now on, we do not restrict anymore the character of the determinants of the 2RDM and 3RDM. As mentioned above, this corresponds to a CASCI using the lowest 20 spin orbitals. This yields a numerically exact ground-state wave function, from which then the initial prefactors $B_{J^{2}, L^{2}}(t=0)$ of the $2 \mathrm{RDM}$ are calculated. Additionally, $\Psi_{\mathrm{GS}}^{\mathrm{CASCI}}$ serves as the initial state of a TD-CASCI calculation, for comparison. A population analysis shows, that the wave-function propagations are extremely close to a FCI result.

The upper panel of Fig. 3 compares the position expectation value. Obviously, the differences begin to appear much earlier now. And just as in the case of CIS, the energy expectation value (lower panel) is a much more sensitive indi-

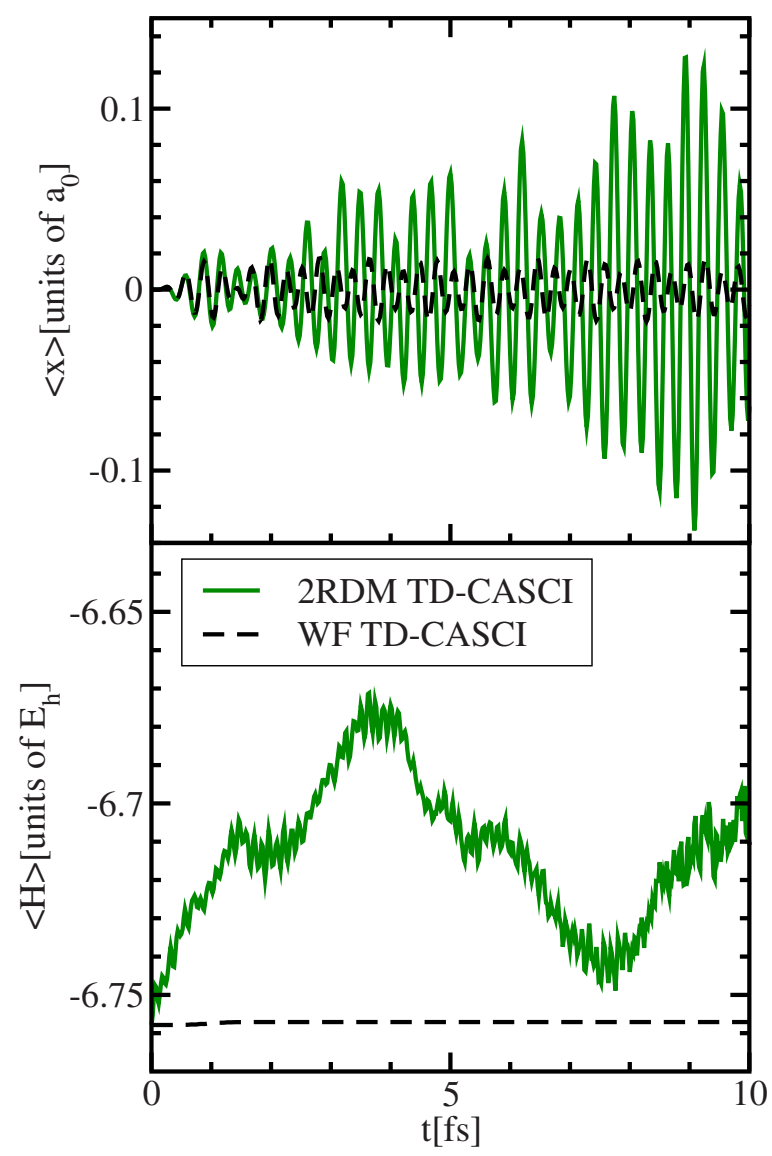

FIG. 3. (Color online) Upper panel: Time-dependent position expectation value for 2RDM (solid line) and wave-function approach (dashed line) using the full CASCI space of the lowest 20 spin orbitals. The lower panel shows the corresponding timedependent energy.

cator that something is going wrong. Therefore, we must conclude that the more correlation is present, the more unstable the propagation becomes.

\section{CONCLUSIONS AND OUTLOOK}

We conclude that the $2 \mathrm{RDM}$ approach to electron dynamics is burdened with an only approximate determination of $d \hat{\rho}^{(2)} / d t$, and a nonlinear equation of motion that leads to a fast growth of errors. If certain limitations are imposed, like the restriction to single excitations only, then propagations of the order of $10 \mathrm{fs}$ are possible, depending on the energy in the system. Of course, this limitation must be overcome, if the 2RDM shall become a useful tool sometime in the future. Our way to truncate the hierarchy of equations of motion and to reconstruct the $3 \mathrm{RDM}$ was based on the most obvious choice for this task. Other, more sophisticated reconstruction functionals have already been developed [28,31-34]. It remains to be seen whether these can perform better than the present approach.

\section{ACKNOWLEDGMENTS}

The authors thank the Deutsche Forschungsgemeinschaft 
for financial support through Grant No. NE 873/2-1.

\section{APPENDIX: DERIVATION OF $B_{J^{2}, L^{2}}$ FROM DOUBLE-HOLE} FUNCTIONS

From Eqs. (2), (5), and (6) we know, that

$$
\begin{aligned}
B_{j_{i} j_{k} l_{m} l_{n}}= & \left\langle\Psi_{l_{m} l_{n}}^{\mathrm{DHF}} \mid \Psi_{j_{j} j_{k}}^{\mathrm{DHF}}\right\rangle \\
= & (2 !)^{2} \sum_{J_{i, k}^{N-2}, L_{m, n}^{N-2}}^{\text {ord }}(-1)^{i+k-3}(-1)^{m+n-3} A_{L^{N}}^{*} A_{J^{N}} \\
& \times\left\langle\operatorname{det}\left(L_{m, n}^{N-2}\right) \mid \operatorname{det}\left(J_{i, k}^{N-2}\right)\right\rangle,
\end{aligned}
$$

where $J_{i, k}^{N-2}$ is created from $J^{N}$ by omitting $j_{i}$ and $j_{k}$ and leaving the order unchanged. Using the antisymmetry of the
$A$ coefficients and tracing out the electrons 3 through $N$ lead to

$$
\begin{aligned}
B_{j_{i} j_{k}, l_{m} l_{n}} & =(2 !)^{2} \sum_{J_{i, k}^{N-2}, L_{m, n}^{N-2}}^{\text {ord }} A_{l_{m} l_{n} L_{m, n}^{N-2} A_{j_{i} j_{k}} J_{i, k}^{N-2}\left\langle\operatorname{det}\left(L_{m, n}^{N-2}\right) \mid \operatorname{det}\left(J_{i, k}^{N-2}\right)\right\rangle}^{* \text { ord }} \\
& =(N-2) !(2 !)^{2} \sum_{J_{i, k}^{N-2}}^{*} A_{l_{m} l_{n} J_{i, k}^{N-2} A_{j_{i} j_{k}} J_{i, k}^{N-2} .}^{*}
\end{aligned}
$$

Again, due to the antisymmetry of the $A$ coefficients the summation can be extended to all $N-2$ tuples. Replacing $j_{i} j_{k}$ by $J^{2}, l_{m} l_{n}$ by $L^{2}$, and $J_{i, k}^{N-2}$ by $K^{N-2}$ we obtain

$$
B_{J^{2}, L^{2}}=(N-2) !(2 !)^{2} \sum_{K^{N-2}}^{\text {ord }} A_{L^{2} K^{N-2}}^{*} A_{J^{2}, K^{N-2}} .
$$

[1] M. Hentschel, R. Kienberger, C. Spielmann, G. A. Reider, N. Milosevic, T. Brabec, P. Corkum, U. Heinzmann, M. Drescher, and F. Krausz, Nature (London) 414, 509 (2001).

[2] H. Niikura, F. Legare, R. Hasbani, A. D. Bandrauk, M. Y. Ivanov, D. M. Villeneuve, and P. B. Corkum, Nature (London) 417, 917 (2002).

[3] A. Baltuska, T. Udem, M. Uiberacker, M. Hentschel, E. Goulielmakis, C. Gohle, R. Holzwarth, V. S. Yakovlev, A. Scrinzi, T. W. Hansch, and F. Krausz, Nature (London) 421, 611 (2003).

[4] R. Kienberger, E. Goulielmakis, M. Uiberacker, A. Baltuska, V. Yakovlev, F. Bammer, A. Scrinzi, T. Westerwalbesloh, U. Kleineberg, U. Heinzmann, M. Drescher, and F. Krausz, Nature (London) 427, 817 (2004).

[5] M. F. Kling, C. Siedschlag, A. J. Verhoef, J. I. Khan, M. Schultze, T. Uphues, Y. Ni, M. Uiberacker, M. Drescher, F. Krausz, and M. J. J. Vrakking, Science 312, 246 (2006).

[6] R. Kienberger, M. Uiberacker, E. Goulielmakis, A. Baltuska, M. Drescher, and F. Krausz, J. Mod. Opt. 52, 261 (2005).

[7] E. Goulielmakis, V. S. Yakovlev, A. L. Cavalieri, M. Uiberacker, V. Pervak, A. Apolonski, R. Kienberger, U. Kleineberg, and F. Krausz, Science 317, 769 (2007).

[8] M. Okunishi, K. Shimada, G. Prümper, D. Mathur, and K. Ueda, J. Chem. Phys. 127, 064310 (2007).

[9] E. Runge and E. K. U. Gross, Phys. Rev. Lett. 52, 997 (1984).

[10] F. Calvayrac, P. G. Reinhard, E. Suraud, and C. A. Ullrich, Phys. Rep. 337, 493 (2000).

[11] T. Kunert and R. Schmidt, Eur. Phys. J. D 25, 15 (2003).

[12] E. Livshits and R. Baer, J. Phys. Chem. A 110, 8443 (2006).

[13] T. Klamroth, Phys. Rev. B 68, 245421 (2003).

[14] P. Krause, T. Klamroth, and P. Saalfrank, J. Chem. Phys. 123, 074105 (2005).

[15] P. Krause, T. Klamroth, and P. Saalfrank, J. Chem. Phys. 127,
034107 (2007).

[16] M. Nest and T. Klamroth, Phys. Rev. A 72, 012710 (2005).

[17] M. Nest, P. Ramanathan, and P. Saalfrank, J. Chem. Phys. 126, 214106 (2007).

[18] F. Remacle, M. Nest, and R. D. Levine, Phys. Rev. Lett. 99, 183902 (2007).

[19] T. Kato and H. Kono, Chem. Phys. Lett. 392, 533 (2004).

[20] J. Caillat, J. Zanghellini, M. Kitzler, O. Koch, W. Kreuzer, and A. Scrinzi, Phys. Rev. A 71, 012712 (2005).

[21] A. I. Kuleff, J. Breidbach, and L. S. Cederbaum, J. Chem. Phys. 123, 044111 (2005).

[22] P. O. Löwdin, Phys. Rev. 97, 1474 (1955).

[23] J. E. Mayer, Phys. Rev. 100, 1579 (1955).

[24] R. H. Tredgold, Phys. Rev. 105, 1421 (1957).

[25] A. J. Coleman and V. I. Yukalov, Reduced Density Matrices: Coulson's Challenge (Springer, New York, 2000).

[26] A. J. Coleman, Rev. Mod. Phys. 35, 668 (1963).

[27] D. A. Mazziotti, Phys. Rev. Lett. 93, 213001 (2004).

[28] P. Ziesche, in Many-Electron Densities and Reduced Density Matrices, edited by J. Cioslowski (Kluwer Academic, New York, 2000).

[29] N. N. Bogolyubov, Vest. Mosk. Univ. Ser. 1: Mat. Mekh 10, 115 (1955).

[30] A. Szabo and N. S. Ostlund, Modern Quantum Chemistry (Dover, New York, 1989).

[31] D. A. Mazziotti, Phys. Rev. A 57, 4219 (1998).

[32] C. Valdemoro, Phys. Rev. A 45, 4462 (1992).

[33] K. Yasuda and H. Nakatsuji, Phys. Rev. A 56, 2648 (1997).

[34] F. E. Harris, Int. J. Quantum Chem. 90, 105 (2002).

[35] A. J. Coleman and I. Absar, Int. J. Quantum Chem. 18, 1279 (1980).

[36] R. Grobe and J. H. Eberly, Phys. Rev. A 48, 4664 (1993).

[37] F. Colmenero and C. Valdemoro, Phys. Rev. A 47, 979 (1993). 